\title{
Quantitative trait loci (QTLs) detection for plant regeneration ability from seed culture in rice (Oryza sativa $\mathrm{L}_{\text {.) }}$
}

\author{
Meihan Liu · Jae-keun Sohn
}

Received: 17 July 2012 / Accepted: 31 July 2012

(c) Korean Society for Plant Biotechnology

\begin{abstract}
Quantitative trait loci (QTLs), which were related to the ability of callus induction and plant regeneration in seed culture of rice, were analyzed using a mapping population from a cross between the rice cultivars 'Samgang' (tongil type) and 'Nagdong' (japonica). A tongil type rice cultivar, 'Samgang' showed lower frequency (20\%) of plant regeneration than that (35\%) of japonica rice, 'Nagdong'. Transgressive segregations were observed for the ability of callus induction and plant regeneration from the seedderived calli of 58 doubled haploid (DH) lines. The ability of plant regeneration of 58 doubled haploid lines showed a continuous distribution with comparatively wide range (10.0 to $66.7 \%$ ) of variation. Composite interval mapping analysis was used to identify the QTLs controlling callus induction and plant regeneration ability. Four significant QTLs, qCWS6, qCWS8, qCWS9 and qCWS11, associated with callus weight per seed were detected on chromosomes 6,8 , 9, and 11 with LOD values of 3.30, 2.60, 2.70 and 2.43, explaining $36 \%$ of the total phenotypic variation. Three significant QTLs, qPR1, qPR6, and qPR11, for the ability of plant regeneration were located on chromosome 1,6 , and 11 at LOD score of 2.25, 2.15 and 2.55, accounting for $24 \%$ of the total phenotypic variation. The present study should be useful for improving the efficiency of plant regeneration in tissue culture of indica rice by means of marker-assisted selection.
\end{abstract}

\section{Introduction}

Crop improvement is based on the manipulation of genetic potential through cross breeding of diverse parents and

M. Liu · J.-K. Sohn $(\bowtie)$

Department of Agronomy, Kyungpook National University, Daegu, 702-701, Korea

e-mail: jhsohn@knu.ac.kr selection in the segregating population. Innovative in vitro approaches that could be incorporated into plant breeding programs include anther (microspore) culture, ovary culture, in vitro fertilization, embryo culture, regeneration from cell culture, and protoplast fusion. The ability of plant regeneration from seed-derived callus of rice is influenced not only by physiological factors (such as media composition, cultural methods and cultural environments), but also by genotypes. Among these factors, the genotype of donor plants is a strong determinant of the regeneration ability from seed callus, and this character is under genetic control (Henry et al. 1994). Several genetic studies have been performed to improve the regeneration ability from seed-derived calli in rice (Peng and Hodges 1989; Taguchi et al. 1996). However, the ability of plant regeneration is quantitatively inherited and greatly affected by the environment, which has made it difficult to improve plant regeneration by selection of highly responsive genotypes, since the genetic basis of tissue culture responses has not been investigated in detail (Krakowsky et al. 2006). Genetic studies on tissue-culture traits, such as callus growth, embryogenesis, and differentiation, will make it possible to transfer genes controlling desirable tissue-culture traits into recalcitrant cultivars or species (Mano and Komatsuda 2002). With advances in molecular biology, traditional quantitative genetics have progressed to the stage of molecular quantitative genetics, thus providing a new route to study complex quantitative traits (Khush and Brar 2004). The use of molecular markers has enabled the identification of quantitative trait loci (QTLs) involved in the expression of important agronomic traits. Furthermore, the construction of genetic maps has provided a tool for identification of the number, significance and location of QTL (Bolibok et al. 2007; Flores Berrios et al. 2000). Recently, DNA markers were used to identify the genes contributing to plant regeneration in maize (Beaumont et al. 1995; Murigneux et al. 1994), tomato (Koornneef et al. 1993), and barley (Mano 
et al. 1996). In rice, Taguchi et al. (1997) mapped five QTLs that are associated with plant regeneration from seed-derived calli on chromosomes 1,2 , and 4 using $\mathrm{BC}_{1} \mathrm{~F}_{5}$ lines from across between cultivars 'Kasalath (indica)' and 'Nipponbare (japonica)'. Takeuchi et al. (2000) identified a QTL for plant regeneration from seed-derived calli in rice on chromosome 2 using the $F_{2}$ population from across between 'Norinl (japonica)' and 'Tadukan (indica)'.

Doubled haploid (DH) populations are useful in the breeding of rice (Oryza sativa L.), and many elite lines have been produced within a short time by the anther culture of $F_{1}$ plants (Zapata et al. 1991; Fang 1991). DH populations are also useful for genetic analysis, particularly for quantitative traits (Snape et al. 1984), and QTLs in rice have been mapped using DH populations (Huang et al. 1996; Lu et al. 1996).

Here we tried to detect QTLs that affect plant regeneration ability (which is included in tissue culture performance) using DH populations derived from a cross between the cultivars 'Samgang' and 'Nagdong'. The objectives of this study were as follows: 1) to evaluate tissue culture performance more precisely, the procedures were divided into two steps: callus induction from seeds, and plant regeneration from induced calli; 2) to demonstrate the existence of QTLs that influence plant regeneration, [detection was attempted].

\section{Materials and methods}

Plant material

One hundred and twenty 'Samgang' and 'Nagdong' doubled haploid (SNDH) lines developed by an anther culture of $\mathrm{F}_{1}$ from across between the cultivars 'Samgang' and 'Nagdong' were used as the mapping population in this study. The DH population was developed through the anther culture described by Kim and Sohn (2005). 'Samgang' is a tongil type cultivar with a large number of grains, heavy panicle and high yield, while 'Nagdong' is a japonica cultivar which is characterized by the good quality and plentiful panicles per plant (Qin et al. 2007). The DH population was cultivated in 2007 and 2008, and was evaluated in a randomized complete block design with two replications. The germinated seeds were sown in the seedbed, and the seedlings were transplanted into three-row plots after 30days. At least seventeen plants were cultivated in each row, with $30 \mathrm{~cm}$ between rows and $15 \mathrm{~cm}$ between plants with in each row. The parents, 'Samgang' and
'Nagdong', as the control lines were cultivated each for ten plots. The brown rices of fifty-eight $\mathrm{DH}$ lines were used in this study.

Callus induction

The mature and dehusked seeds of the SNDH population were washed with $70 \%$ ethanol for 30 seconds, then with $1 \%$ sodium hypochlorite solution for 40 minutes in a shaking incubator, and finally rinsed with sterile water through mild shaking three times. Ten of the sterilized seeds were plated onto one Petri dish $(90 \times 15 \mathrm{~mm})$ containing $20 \mathrm{ml}$ of callus induction medium composed of Murashige and Skoog (MS) medium (Murashige \& Skoog, 1962) supplemented with $2 \mathrm{mg} / \mathrm{L}$ 2,4-dichlorophenoxyacetic acid (2,4-D), $30 \mathrm{~g} / \mathrm{L}$ sucrose, $2 \mathrm{mg} / \mathrm{L}$ casein hydrolysate, $5 \mathrm{~g} / \mathrm{L}$ gelrite, and adjusted to $\mathrm{pH} 5.8$ before autoclaving. The brown rices cultured in Petri dishes were kept under dark condition at $26^{\circ} \mathrm{C}$ for 3 weeks to allow callus formation. The callus induction rate was evaluated as the proportion of the calli-forming seeds to the total number of cultured seeds. The weights of the calli were measured at five weeks after the culture of brown rices on callus induction medium.

\section{Plant regeneration}

After three weeks, the calli were transferred to plant regeneration medium in a $90 \times 15 \mathrm{~mm}$ sized Petri dish $(25 \mathrm{ml}$ per Petri dish). The regeneration medium was MS basal medium supplemented with $1 \mathrm{mg} / \mathrm{L}$ naphthalene acetic acid (NAA), $5 \mathrm{mg} / \mathrm{L}$ kinetin, $5 \mathrm{~g} / \mathrm{L}$ gelrite and also adjusted to pH 5.8 before autoclaving. Thirty Petri dishes, each of which had ten seeds or calli, were cultured for each experimental group under fluorescent lamps for 45 days. The frequency of plant regeneration was calculated as the number of calli with a regenerated shoot among the total number of calli transferred onto the regeneration medium.

\section{QTL analysis}

Prior to the QTL analysis, the percent data were transformed using statistical analysis. The QTLs associated with plant regeneration and callus weight were analyzed using the composite interval mapping analysis of the computer program, WinQTL Cartographer 2.5 (Basten et al. 2005; Zeng, 1994). A LOD score of 2.0 was used as the significant threshold for QTL locations. The nomenclature of the QTLs was adopted from McCouch et al. (1997). 
Two to five lowercases were used to denote the trait actually measured. A number was added to the end of the locus name to designate the rice chromosome (1-12) on which it had been mapped to. If more than one QTL for the same trait was detected on the same chromosome in the present study, then a second numerical identifier was added \{i.e., [a comma (,) and the new numerical identifier] following the chromosomal designation $\}$ to differentiate the individual QTL.

\section{Results and discussion}

Phenotypic variation of parents and $58 \mathrm{DH}$ lines

This section shows the phenotypic variation among parents and $58 \mathrm{DH}$ lines. The tissue culture performance of the cultivar 'Samgang' was much lower than that of the cultivar 'Nagdong'. Table 1, Figure 1 shows callus induction and plant regeneration from the seed culture of the parental cultivars 'Samgang' and 'Nagdong' in rice. The calli derived from 'Samgang' turned to a brown colour at forty days after the seed culture, while the calli from 'Nagdong' grew well.

The callus weight from 'Samgang' was $63.1 \mathrm{mg}$ and from 'Nagdong' was $94.6 \mathrm{mg}$. The callus weight for SNDH population ranged from $15.0 \mathrm{mg}$ to $195.6 \mathrm{mg}$ with continuous variation (Fig. 2A). The rate of plant regeneration from 'Samgang' and 'Nagdong' was 20\% and 35\%, respectively. The rate of plant regeneration of SNDH population showed a continuous distribution with wide range (10.0 to $66.7 \%$ ) of variation (Fig. 2B).

A normal distribution is an indication of polygenic inheritance and suggests the existence of some alleles, which promotes that not all the alleles with positive effects occur in the same parent. In this study, 'Nagdong' might be the allele, which promotes the $\mathrm{DH}$ lines in vitro regeneration, and 'Samgang' might inhibit the DH lines in vitro.

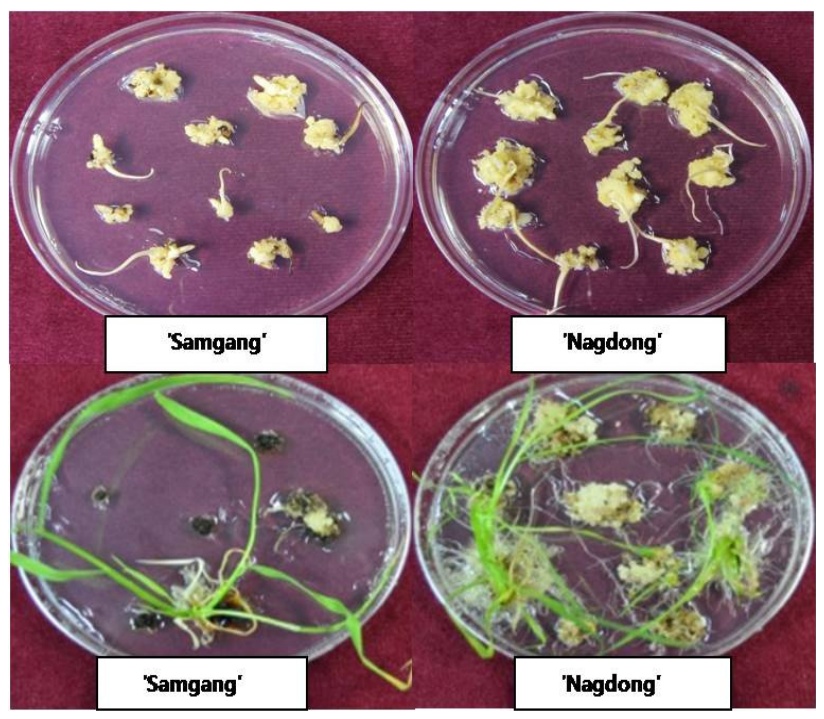

Fig. 1 Callus induction and plant regeneration from seed calli derived from the parental cultivars, 'Samgang' and 'Nagdong'

Table 1 Analysis of variance for traits in 'Samgang' and 'Nagdong' cultivars and DH population

\begin{tabular}{|c|c|c|c|c|c|c|}
\hline \multirow{2}{*}{ Cultivars } & \multicolumn{2}{|c|}{ Callus Induction } & \multicolumn{2}{|c|}{ Callus Weight } & \multicolumn{2}{|c|}{ Plant Regeneration } \\
\hline & Mean & Range & Mean & Range & Mean & Range \\
\hline Samgang & $81.48 \pm 9.31$ & $58.70-96.30$ & $88.95 \pm 28.39$ & $33.04-155.93$ & $28.58 \pm 17.28$ & $0-100.00$ \\
\hline Nagdong & $74.86 \pm 17.11$ & $30.00-99.00$ & $96.43 \pm 38.65$ & $33.33-193.89$ & $24.08 \pm 15.26$ & $0-67.50$ \\
\hline
\end{tabular}
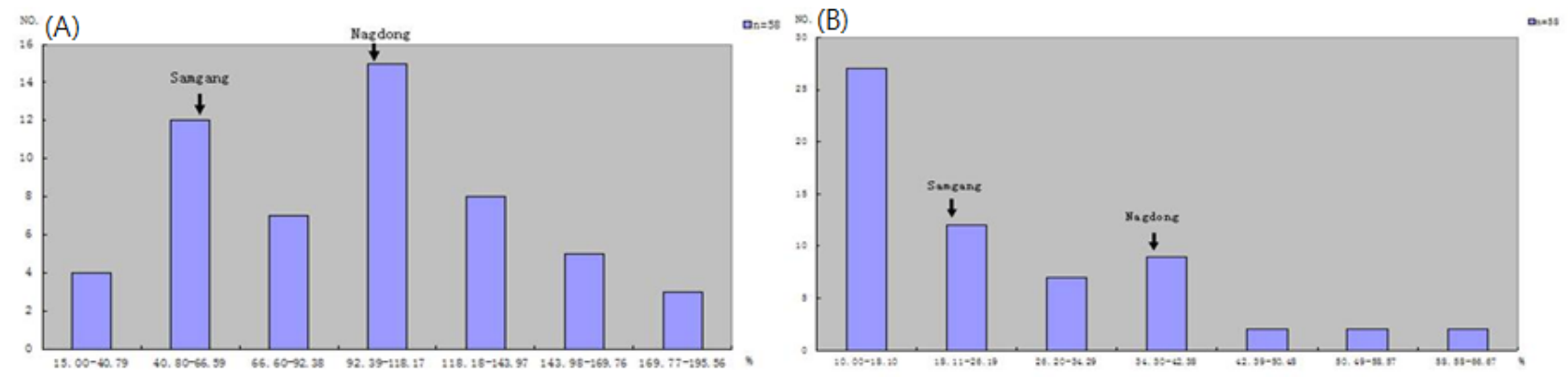

Fig. 2 Frequency distribution of the rate from seed culture of $58 \mathrm{DH}$ lines in rice; (A) callus weight per seed, (B) the rate of plant regeneration from the seed-derived calli. 
QTLs for callus weight and plant regeneration

In total, four significant QTLs, qCWS6, qCWS8, qCWS9 and qCWS11, associated with callus weight, were detected on chromosome 6, 8, 9, and 11 (Fig. 3). The LOD scores were $3.30,2.60,2.70$ and 2.43, respectively. Three significant QTLs, qPR1, qPR6 and qPR11, related to plant regeneration were detected on chromosome 1,6 and 11 . Their LOD scores were 2.25, 2.15 and 2.55, respectively. The qCWS6 and qCWS8 detected with callus weight were
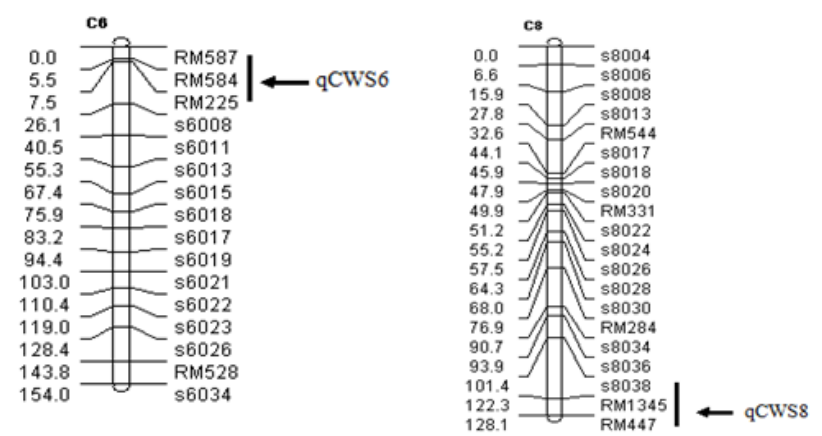

(B)

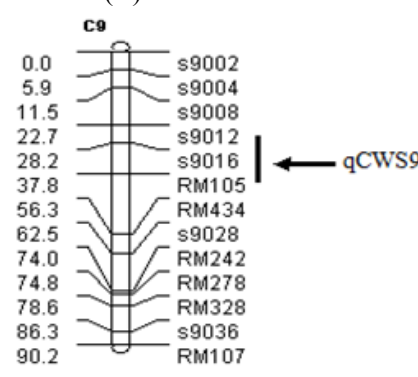

(C)

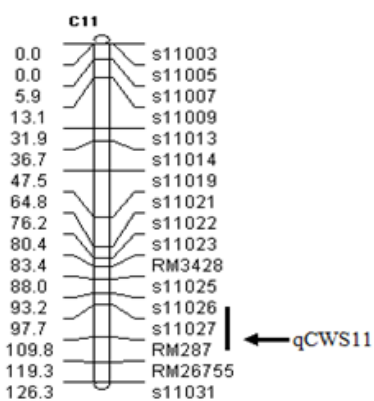

(D)

Fig. 3 Chromosome location of QTLs related to callus weight per seed (A, B, C and D) mapped in the intervals RM587-RM225 and s8038-RM447 on chromosome 6 and 8, respectively (Fig. 3A, B). The qCWS9 was located on chromosome 9 between markers s9012 and RM105 (Fig. 3C), and qCWS11 on chromosome 11 between markers s11026 and RM287 (Fig. 3D).

The qPR1 associated with plant regeneration was detected on chromosome 1 between markers s1028 and s1030 (Fig. 4A). The qPR6 was located on chromosome 6 between markers s6013 and s6018 (Fig. 4 B), and qPR11 on chromosome 11 between markers s11026 and RM287 (Fig. 4C).

On the basis of the composite interval mapping analysis, four QTLs, qCWS6, qCWS8, qCWS9, and qCWS11, associated with callus weight explained $36 \%$ of the total phenotypic variation, which in the alleles of 'Nagdong' increased the callus weight with an additive effect of 19.70, 17.20, 13.86 and 20.00. Three QTLs, qPR1, qPR6 and qPR11, for plant regeneration accounted for $24 \%$ of total phenotypic variation. These QTLs in the alleles of 'Nagdong' increased the ability of plant regeneration with the additive effect from 4.94 to 6.67 (Table 2).

In this study, we mapped four QTLs related to callus weight and three QTLs related to plant regeneration in the seed culture of $58 \mathrm{DH}$ population derived from a cross between 'Samgang' and 'Nagdong'. Three QTLs associated with plant regeneration ability were not likely to correspond to the gene locations of three QTLs associated with callus weight. The two parents, 'Samgang' and 'Nagdong', have different phenotypes for regeneration ability (Fig. 1), and the difference for regeneration between the parents is clear. Moreover, three QTLs associated with plant regeneration explained $24 \%$ for phenotypic variance. These results indicated a complex inheritance of seed culture ability that may be controlled by multiple genes. The present study was also

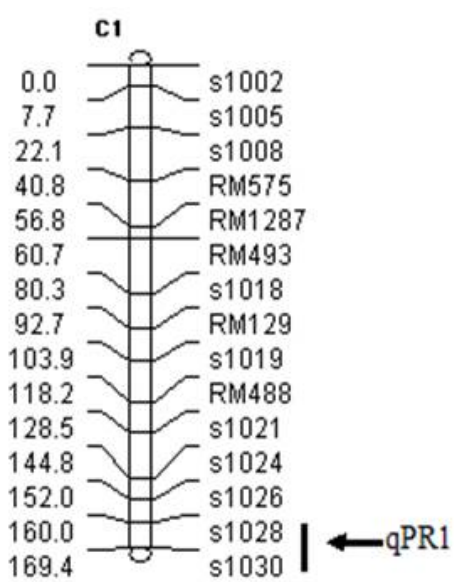

(A)

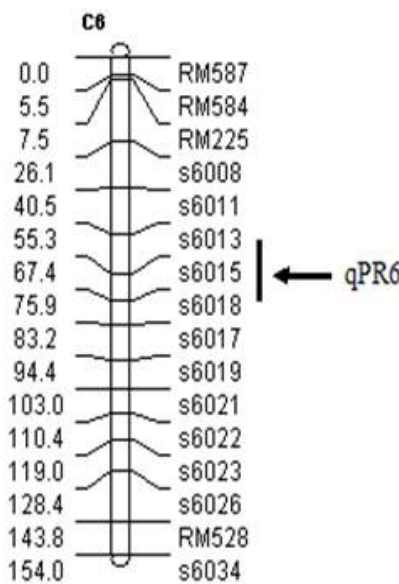

(B)

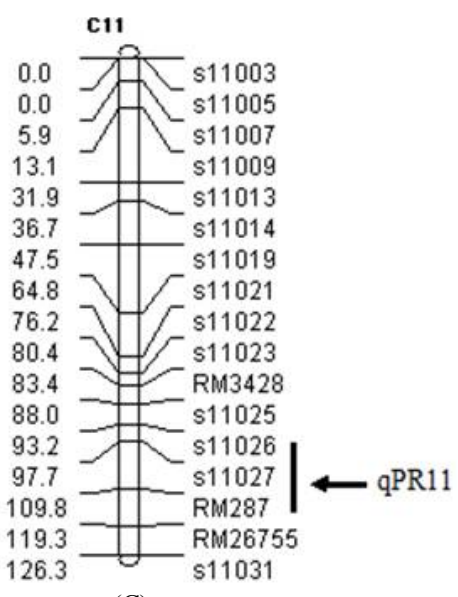

(C)

Fig. 4 Chromosome location of QTLs related to plant regeneration (A, B and C) 
Table 2 Characteristics of QTLs associated with callus weight and plant regeneration in the seed culture of the SN DH population in rice

\begin{tabular}{|c|c|c|c|c|c|c|}
\hline Traits & QTLs & Chromosome & Bordering markers & LOD & $\mathrm{R}^{2}(\%)$ & Additive effect \\
\hline \multicolumn{7}{|c|}{ Callus weight } \\
\hline & qCWS6 & 6 & RM587-RM225 & 3.30 & 11 & -19.70 \\
\hline & qCWS8 & 8 & s8038-RM447 & 2.60 & 8 & -17.20 \\
\hline & qCWS9 & 9 & s9012-RM105 & 2.70 & 8 & -13.86 \\
\hline & qCWS11 & 11 & ss11026-RM287 & 2.43 & 9 & -20.00 \\
\hline Total phe & variation & TLs & & & 36 & \\
\hline \multicolumn{7}{|c|}{ Regeneration rate } \\
\hline & qPR1 & 1 & s1028-s1030 & 2.25 & 9 & -6.65 \\
\hline & qPR6 & 6 & s6013-s6018 & 2.15 & 7 & -4.94 \\
\hline & qPR11 & 11 & s11026-RM287 & 2.55 & 8 & -6.67 \\
\hline \multicolumn{5}{|c|}{ Total phenotypic variation of 3 QTLs } & 24 & \\
\hline
\end{tabular}

$\mathrm{R}^{2}$ : percentage of phenotypic variation explained;

Additive effect, '-' indicates alleles from 'Nagdong'.

LOD: logarithm of odds.

able to prove such complexity. Three new QTLs, qPR1, qPR6, and qPR11, contributing to plant regeneration in this study were located on chromosome 1, 6, and 11 which were different from that of Kwon et al. (2000), showing negative additive effects. All the negative additive effects showed the alleles were from 'Nagdong'. However, these QTLs also were associated with the regeneration ability from the seed-derived callus of rice. The mismatch of locations of QTLs for plant regeneration mapped by Taguchi et al. (1997) and Takeuchi et al. (2000) may be related to the use of a different genetic background of the mapping population. Different population such as $F_{2}$ population, $\mathrm{DH}$ and recombinant inbred lines used in genetic mapping of QTLs for tissue culture regeneration, could yield notable discrepancy in the genetic loci identified (Zhao et al. 2008).

In the present study, three QTLs associated with plant regeneration from the seed-derived callus showed there was one or more genes for increasing regeneration ability of SNDH lines in rice (Table1). Though QTL detection for plant regeneration ability have also been done for numerous plant species such as barley and maize, it is not yet clear whether there are any genes in common in different species. In maize, Murigneux et al. (1994) reported that QTLs controlling androgenesis were obviously genotypedependent and only representative of the differences between the parents. The objective of QTL mapping in this study is to utilize the trait to improve the indica type rice tissue culture ability. An effective method to achieve this is the use of molecular markers linked the QTLs. In addition, comparative mapping of genera and/or families using common markers in the vicinity of such QTL is necessary to identify homologous QTL or genes which can work universally in plant tissue culture (Taguchi et al. 1997). To identify such QTLs and improve plant regeneration ability, genetic transformation with DNA markers might has been suggested in numerous studies. In a word, the utilization of DNA markers that are tightly linked to these QTLs should help for the selection of genotypes with high regeneration ability and help in the transfer of the regeneration ability to genotypes that respond poorly (Kim et al. 1999). Moreover, our results should also help to identify genes related to plant regeneration from seedderived calli in rice. Future studies will be directed toward identification and marker-assisted selection of genes associated with these QTLs.

\section{Acknowledgement}

This work was supported by a grant from the NextGeneration BioGreen 21 Program (No. PJ00806201), Rural Development Administration, Republic of Korea.

\section{References}

Basten CJ, Weir BS, Zeng ZB (2005) QTL Cartographer version 1.17

Beaumont VH, Rocheford T, Widholm JN (1995) Mapping the anther culture response genes in maize (Zea may $L$.). Genome 38:968-975

Bolibok H, Gruszczynska A, Hromada-Judycka A, Rakoczy- 
Trojanowska M (2007) The identification of QTLs associated with the in vitro response of rye (Secale cereale L.). Cell. Mol. Biol. Lett. 12:523-535

Fang PY (1991) Breeding new rice strains through anther culture. In: Y.P.S Bajaj (Ed), Biotechnology in agriculture and forestry. Springer-Verlag, Berlin, Heidelberg Vol 14: Rice pp 216-229

Flores Berrios EL, Sarrafi A, Fabre F, Alibert G, Gentzbittel L (2000) Genotypic variation and chromosomal location of QTLs for somatic embryogenesis revealed by epidermal layers culture of recombinant inbred lines in the sunflower (Helianthus annuus L.). Theor Appl Genet 101:1307-1312

Henry Y, Vain P and De Buyser J (1994) Genetic analysis of in vitro plant tissue culture responses and regeneration capacities. Euphyrica 79:45-58

Huang N, Courtois B, Khush GS, Lin H, Wang G, Wu P. \& Zheng K (1996) Association of quantitative trait loci for plant height with major dwarfing genes in rice. Heredity 77:130-137

Khush GS, Brar DS (2004) Harnessing science and technology for sustainable rice-based production systems (English). In : Proceeding of the FAO Rice Conference; International Rice Conference, Rome (Italy), 12-13 Feb 2004/FAO, Rome (Italy). Plant Production and Protection Div. 17-23

Kim KM, Park GH, Kim JH, Kwon YS, Sohn JK (1999) Selection of RAPD marker for growth of seedlings at low temperature in rice. Mol. Cells 9, 265-269

Kim SM, Sohn JK (2005) Identification of a rice gene (Bph 1) conferring resistance to brown planthopper (Nilaparvata lugens Stal) using STS markers. Mol Cells 20:30-34

Koornneef M, Bade J, Hanhart C, Horsman K, Schel J, Soppe W, Verkerk R, Zabel P (1993) Characterization and mapping on a gene controlling shoot regeneration in tomato. Plant $\mathrm{J}$ 3:131-141

Krakowsky MD, Lee M, Garay L, Woodman-Clikeman W, Long MJ, Sharopova N, Frame B, Wang K (2006) Quantitative trait loci for callus initiation and totipotency in maize (Zea mays L.). Theor Appl Genet 113:821-830

Kwon YS, Kim KM, Eun MY, Sohn JK (2000) Quantitative trait loci mapping associated with plant regeneration ability from seed derived calli in rice (Oryza sativa L.) Mol Cells 11:64-67

Kwon YS, Kim KM, Eun MY, Sohn JK (2002) QTL mapping and associated marker selection for the efficacy of green plant regeneration in anther culture. Plant Breed 12:10-16

Lu C, Shen L, Tan Z, Xu Y, He P, Chen Y, Zhu L (1996) Comparative mapping of QTLs for agronomic traits of rice across environment using a doubled haploid population. Theor Appl Genet 93:1211-1217
Mano Y, Komatsuda T (2002) Identification of QTLs controlling tissue-culture traits in barely (Hordeum vulgare L.). Theor Appl Genet 105:708-715

Mano Y, Takahashi H, Sato K, Takeda K (1996) Mapping genes for callus growth and shoot regeneration in barley. Breeding Science 46:137-142

McCouch SR, Cho YG, Yano M, Paul E, Blinstrubb M, Morishima H, Kinoshita T (1997) Report on QTL nomenclature. Rice Genet New 14:11-13

Murashige T, Skoog F (1962) A revised medium for rapid growth and bioassays with tobacco tissue cultures. Physiol Plant $15: 473-497$

Murigneux A, Bentolila S, Hardy T, Baud S, Guitton C, Jullien H, Ben Tahar S, Freyssinet G, Beckert M (1994) Genotypic variation of quantitative trait loci controlling in vitro androgenesis in maize. Genome 37:970-976

Peng J, Hodges T (1989) Genetic analysis of plant regeneration in rice (Oryza sativa L.). In vitro Cell Dev Biol 25:91-94

Qin Y, Kim SM, Sohn JK (2007) Genetic analysis for rice grain traits using a doubled haploid population. Korean J. Crop Sci 52:123-128

Snape JW, Wright AJ, Simpson E (1984) Methods for estimating gene numbers for quantitative characters using doubled haploid lines. Theor Appl Genet 67:143-148

Taguchi SF, Komatsuda T, Oka S (1996) Comparison of two indices for evaluating regeneration ability in ride (Oryza sativa L.) through a diallel analysis. Theor Appl Genet 94:378-382

Taguchi SF, Lin SY, Tanno K, Komatsuda T, Yano M, Sasaki T, Oka S (1997) Mapping quantitative trait loci associated with regeneration ability of seed callus in rice, Oryza sativa L. Theor Appl Genet 95:828-833

Takeuchi Y, Abe T, Sasahara T (2000) RFLP mapping of QTLs influencing shoot regeneration from mature seed-derived calli in rice. Crop Sci 40:245-247

Zapata FJ, Alejar MS, Torrizo LB, Novero AU, Singh VP Senadhira D (1991) Field performance of anther-culturederived lines from $\mathrm{F}_{1}$ crosses of indica rices under saline and monsaline conditions. Theor Appl Genet 83:6-11

Zeng ZB (1994) Precision mapping of quantitative trait loci. Genetics 136:1457-68

Zhao LN, Zhou HJ, Lu LX, Liu L, Li XH, Lin YJ, Yu SB (2008) Identification of quantitative trait loci controlling rice mature seed culturability using chromosomal segment substitution lines. Plant Cell Rep 28:247-256 